\title{
Favourable effects of Bacillus subtilis and Bacillus licheniformis on experimental periodontitis in rats
}

\author{
Michel R. Messora ${ }^{\mathrm{a}, *}$, Luciano J. Pereira ${ }^{\mathrm{b}}$, Renata Foureaux ${ }^{\mathrm{b}}$, Luiz F.F. Oliveira ${ }^{\mathrm{a}}$, \\ Caio G. Sordi ${ }^{a}$, Ana J.N. Alves ${ }^{a}$, Marcelo H. Napimoga ${ }^{c}$, Maria J.H. Nagata ${ }^{\text {, }}$, \\ Edilson Ervolino ${ }^{e}$, Flávia A.C. Furlaneto ${ }^{a}$ \\ a Department of Oral and Maxillofacial Surgery and Periodontology, School of Dentistry of Ribeirao Preto, University of Sao Paulo-USP, Av. Café, \\ 14040-904 Ribeirao Preto, SP, Brazil \\ b Physiology and Pharmacology Department, Federal University of Lavras, Av. Doutor Sylvio Menicucci, 1001, 37200-000 Lavras, MG, Brazil \\ ${ }^{\mathrm{c}}$ Laboratory of Immunology and Molecular Biology, Sao Leopoldo Mandic, Street Dr. José Rocha Junqueira, 13, 13045-755 Campinas, SP, Brazil \\ d Department of Surgery and Integrated Clinic, Dental School of Aracatuba, UNESP-Univ Estadual Paulista, Street José Bonifácio, 1193, 16015-050 Aracatuba, \\ SP, Brazil \\ e Department of Basic Sciences, Division of Histology, Dental School of Aracatuba, UNESP-Univ Estadual Paulista, Rodovia Marechal Rondon, km 527, \\ 16021-900 Aracatuba, SP, Brazil
}

\section{A R T I C L E I N F O}

\section{Article history:}

Received 19 August 2015

Received in revised form 10 December 2015

Accepted 23 February 2016

\section{Keywords:}

Probiotics

Root planing

Periodontitis

Rats

\begin{abstract}
A B S T R A C T
Objectives: The purposes of this study were to evaluate, in rats: i) the effects of Bacillus species on the development of experimental periodontitis (EP) via microtomographic, immunological and hematological assays (Experiment 1-E1); ii) the effects of Bacillus species as adjuncts to scaling and root planing (SRP) for the treatment of EP via histomorphometric and immunohistochemical analyses (Experiment 2-E2).

Methods: In E1, 24 rats were divided into groups C1 (control), PROB1, EP1 and EP-PROB1. In groups with $\mathrm{EP}$, the mandibular first molar of each animal received a ligature for 14 days. In groups PROB1, animals received Bacillus species for 44 days, starting 30 days before EP induction in Group EP-PROB1. In E2, 24 rats were assigned to groups C2 (control), PROB2, EP-SRP2 and EP-SRP-PROB2. In groups with SRP, EP was induced as described in E1. The ligatures were removed after 14 days and SRP was performed. In groups PROB2, animals received Bacillus species for 15 days, starting after SRP in Group EP-SRP-PROB2. Results: In E1, Group EP1 presented bone loss (BL) and eosinophil numbers greater than Group EP-PROB1 $(P<0.05)$. In Group EP-PROB1, the receptor activator of nuclear factor-kB ligand (RANKL)/osteoprotegerin (OPG) ratio was similar to that of groups without EP. In E2, Group EP-SRP-PROB2 presented fewer TRAPpositive osteoclasts, lower immunolabeling pattern for a proinflammatory cytokine and decreased BL and attachment loss than Group EP-SRP2 $(P<0.05)$.

Conclusions: Bacillus species supplementation provided a protective effect against BL and enhanced the effects of SRP in the treatment of EP in rats.
\end{abstract}

(c) 2016 Elsevier Ltd. All rights reserved.

\section{Introduction}

In the past few years, the use of probiotics to displace pathogenic microorganisms in order to maintain or restore a natural microbiome has been considered a promising way to combat diverse infectious processes, including those of the oral cavity (Bengmark, 2001; Stamatova \& Meurman, 2009a, 2009b; Vuotto, Barbanti, Mastrantonio, \& Donelli, 2014). Apparently, this

\footnotetext{
* Corresponding author.

E-mail address: m.messora@forp.usp.br (M.R. Messora).
}

therapy may modify the bacterial environment and modulate the host immune response (Teughels, Loozen, \& Quirynen, 2011). The latter effects are appealing for periodontal healthcare because there is evidence demonstrating that the destruction of periodontal tissues is substantially mediated by the host and driven by the bacterial challenge (Sanz and Quirynen, 2005; Sanz \& van Winkelhoff, 2011).

Probiotics are defined as live microorganisms that can confer health benefits when administered in adequate amounts (FAO/WHO, 2002). The main microorganisms used as probiotics are bacteria from the genera Lactobacillus,Enterococcus, Bacillus and Bifidobacterium (Bron, van Baarlen, \& Kleerebezem, 2012). Teughels 
et al. (2013) and Vivekananda, Vandana, and Bhat (2010) have demonstrated that bacteria from the Lactobacillus genus combined with scaling and root planing (SRP) may improve periodontal clinical parameters and delay recolonization of periodontal pockets with periodontopathogens in patients with chronic periodontitis.

Although promising results have been reported in the scientific literature, it is important to remember that the findings obtained with probiotics cannot be generalized, since they are dependent on the strain, dosage, frequency and route of administration (Teughels et al., 2011). To date, studies that have investigated the effects of probiotics on periodontal diseases used mainly microorganisms from the genus Lactobacillus (Teughels et al., 2013; Vivekananda et al., 2010). To the best of the authors' knowledge, there are no studies that have evaluated the effects of the genus Bacillus as an adjunct to SRP for the treatment of periodontitis.

Scientific interest in spore-forming Bacillus species as probiotics has occurred only in the last few years (Cutting, 2011). Among the species that have been most extensively examined are Bacillus subtilis, Bacillus clausii, Bacillus cereus, Bacillus coagulans and Bacillus licheniformis (Cutting, 2011). Spore probiotics are being widely used in humans as dietary supplements, in animals as growth promoters and competitive exclusion agents and lastly in aquaculture for enhancing the growth and disease-resistance of cultured shrimps (Cutting, 2011). There are three mechanisms by which Bacillus interacts with hosts: stimulation of the immune system, suppression of gastrointestinal pathogens and secretion of antimicrobial compounds (Mongkolthanaruk, 2012).

In previous studies by this group (Foureaux et al., 2014; Messora et al., 2013), the effects of Bacillus species on experimental periodontitis (EP) in rats were evaluated. In the first study, (Messora et al., 2013) it was histologically demonstrated that this supplementation reduced both attachment and alveolar bone losses. In the second study, (Foureaux et al., 2014) Bacillus species decreased the levels of $\mathrm{C}$-terminal telopeptide and increased the expression of osteoprotegerin (OPG) in periodontal tissues. Tsubura et al. (2009) reported that a mouth rinsing prepared from the supernatant of a cultured medium of Bacillus subtilis isolated from Japanese soil improved the gingival index of patients with chronic periodontitis and promoted a marked change in the scores of the benzoyl-DL-arginine-naphthylamide (BANA) test.

However, there is still limited scientific evidence to support the efficacy of Bacillus species in periodontitis. Further studies are essential to improve the scientific basis for their use, elucidate the role of these bacteria in the oral cavity and identify their mechanisms of interaction with the host. The purposes of this study were to evaluate, in rats: i) the effects of Bacillus species on the development of EP via microtomographic, immunological and hematological assays (Experiment 1-E1); ii) the effects of Bacillus species as adjuncts to scaling and root planing (SRP) for the treatment of EP via histomorphometric and immunohistochemical analyses (Experiment 2-E2).

\section{Materials and methods}

\subsection{Sample}

This study was conducted in compliance with the ethical principles of animal experimentation, as well as standards for the didactic-scientific practice of vivisection and the Universal Declaration of Animal Rights by UNESCO (United Nations Educational, Scientific and Cultural Organization). The present study was conducted only after review and approval by the Ethics Committees on Animal Experimentation (ECAE) at School of Dentistry of Ribeirao Preto, University of São Paulo-FORP/USP (protocol 2013.1.993.58.6), at Federal University of Lavras-UFLA (protocol 036/2010) and at Lavras University Center-UNILAVRAS (protocol 012/2010). A power calculation was performed to determine the sample size. The animal was considered the study unit. A sample size was desired that would provide $80 \%$ power to recognize a significant difference of $20 \%$ among groups and a standard deviation of $15 \%$ with a $95 \%$ confidence interval ( $\alpha=0.05)$, considering the change in alveolar bone loss as the primary outcome variable. Therefore, a sample size of six animals per group was required.

Forty-eight adult male Wistar rats (Rattus norvegicus, albinus), weighing 170-250 g, were used (Central Animal Facilities-FORP/USP and UNILAVRAS). The rats were kept in a room with a $12 \mathrm{~h}$ light/dark cycle and temperatures between 22 and $24^{\circ} \mathrm{C}$. The animals were housed in individual metabolic cages and subjected to a period of 7 days of acclimatization to the environment and team running the project. Throughout the experiment, the animals were fed a selected solid diet and water ad libitum. The study was divided into two independent experiments (Fig. 1).

\subsection{Experiment 1-Bacillus species in the development of EP}

In Experiment 1, the effects of Bacillus species on the development of EP were evaluated. 24 rats were randomly assigned to one of four experimental groups $(n=6)$ : C1 (control),

Experiment 1 - Bacillus species in the development of EP

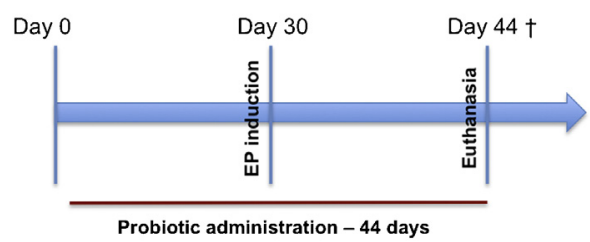

Experiment 2 - Bacillus species in the treatment of EP

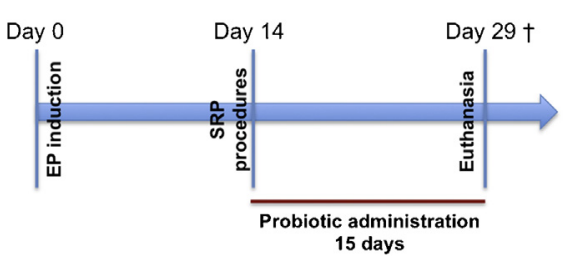

Fig. 1. Experimental design. 
PROB1 (probiotic), EP1 (experimental periodontitis) and EP-PROB1 (experimental periodontitis + probiotic).

\subsubsection{Probiotic therapy, induction of EP and euthanasia}

The probiotic therapy started in the beginning of the experiment (day 0). In Groups PROB1 and EP-PROB1, the probiotic product (Bacillus subtilis-DSM 5750+Bacillus licheniformis-DSM 5749; Bioplus ${ }^{\circledR} 2 B$, Chr. Hansen, Hørsholm, Denmark) was administered orally by adding it to the drinking water of the animals in the proportion of $1.5 \times 10^{8}$ colony-forming units (CFU) per milliliter for the duration of the experiment (44 days). Every morning, $10 \mathrm{~mL}$ of water containing the probiotics was provided to the animals as the unique hydric source. Only after the complete consumption of this initial hydric source, water ad libitum (without probiotics) was given to the animals during the rest of the day. Animals of Groups C1 and EP1 received water without probiotic supplementation.

Thirty days after the beginning of the experiment, all animals were anesthetized by an intraperitoneal injection of xylazine (10 mg/kg body weight; Rompum ${ }^{\circledR}$, Bayer Animal Health, Sao Paulo, SP, Brazil) and ketamine ( $80 \mathrm{mg} / \mathrm{kg}$ body weight; Dopalen ${ }^{\circledR}$, Agribands Purina do Brasil Ltda., Paulinia, SP, Brazil). After general anesthesia, animals of Groups EP1 and EP-PROB1 were placed on the operating table, which allowed the rats' mouths to be kept open, facilitating access to posterior teeth of the mandible. A cotton ligature was placed around the right mandibular first molar of each animal. Cotton ligatures were not placed in the animals of Groups C1 or PROB1.

All animals were euthanized 44 days after the beginning of the experiment by decapitation following recommendations of the ECAE. Prior to decapitation, animals were anesthetized by an intraperitoneal injection of xylazine (Rompum ${ }^{\circledR}$ ) and ketamine (Dopalen ${ }^{\circledR}$ ). $2 \mathrm{~mL}$ of blood, stored in tubes with EDTA, was drawn via cardiac puncture for hematological analysis. Hemimandibles were excised and fixed in $4 \%$ formaldehyde for $48 \mathrm{~h}$ in preparation for microtomographic analysis. Samples of gingival tissues around right mandibular first molars were collected for Western blot analysis.

\subsubsection{Microcomputed tomography (micro-CT) analyses}

Non-demineralized specimens were scanned by a cone-beam micro-CT system (Skyscan 1172, Bruker, Kontich, Belgium). The $\mathrm{X}$-ray generator was operated at an accelerated potential of $60 \mathrm{kV}$ with a beam current of $165 \mu \mathrm{A}$ and an exposure time of $650 \mathrm{~ms}$ per projection. Images were produced with a voxel size of $6 \times 6 \times 6$ $\mu \mathrm{m}$. Appropriate software (Data Viewer ${ }^{\circledR}$, version 1.5.0, Bruker, Kontich, Belgium) was used to generate 3 dimensional models which were then rotated into a standard position according to the following criteria: (1) in the transaxial plane, the right mandibular first molar (M1) had its axis vertically positioned, (2) in the coronal plane, the mandibular bone was vertically orientated, with the mesial root of the M1 in the upper part of the image and (3) in the sagittal plane, the occlusal surface of M1 was horizontally positioned. Linear measurements of alveolar bone level (ABL) were performed at four different sites: buccal, lingual, interproximal and furcation. For buccal and lingual sites, on the transaxial image passing through the distal root of the M1, the linear distances from cementoenamel junction (CEJ) to buccal/lingual alveolar bone crest $(\mathrm{ABC})$ were measured. For interproximal sites, coronal dataset was analyzed using appropriate software (CT-Analyser ${ }^{\circledR}$, version 1.13.5.1+, Bruker, Kontich, Belgium). The distance between the last image showing the $A B C$, between mandibular second molar (M2) and M1, and the first image showing the CEJ of M1, was measured. For the furcation site, on the sagittal image passing through both mesial and distal roots of M1, ABL was assessed by measuring the distance between the roof of the furcation and the $A B C$ in the furcation area. The sum of the four linear measurements obtained from each animal were expressed as the ABL value.

For volumetric measurements, a volume of interest (VOIprismatic section) was outlined from the apexes of all roots of M1 up to the roof of the furcation of M1, touching the roots surfaces, in all images of the coronal dataset, using the same software (CT-Analyser ${ }^{\circledR}$ ) applied for the analysis of the interproximal site. The images were binarised so that bone and dental structures could be distinguished according to differences in the density, using a greyscale (inferior limit-65, superior limit-255; greyscale threshold 0-255). This pattern of binarisation was used for all samples. The following parameters were analyzed: i) Bone Volume (BV): percentage of the VOI filled with bone tissue; ii) Mean Bone Porosity (BP): percentage of bone porosity present in the VOI. All micro-CT analyses were performed by one blinded and calibrated examiner (A.J.N.A.).

\subsubsection{Western blot analysis}

Quantification of receptor activator of nuclear factor-kB ligand (RANKL; SC 7628, Santa Cruz Biotechnology, Santa Cruz, CA, USA) and OPG (SC 11383, Santa Cruz Biotechnology, Santa Cruz, CA, USA) was visualized from total protein extracts of gingival tissues samples. Samples were processed as previously described (Foureaux et al., 2014). Images obtained on radiographic films were scanned by a chemiluminescent documentation system (ECL Prime Western Blotting Detection Reagent-RPN2232, GE Healthcare, Buckinghamshire, UK). Additional analysis of the bands was performed densitometrically using specific software (ImageJ, National Institutes of Health, Washington, DC).

\subsubsection{Hematological analysis}

A blinded technician conducted the hematological analysis immediately after blood collection. Blood samples were processed and analyzed according to the manufacturer's instructions in a calibrated and automated hematology analyzer (ABX Micros ABC Vet, Horiba ABX Diagnostics, Montpellier, France) to obtain counts $\left(10^{3} / \mu \mathrm{L}\right)$ of neutrophils, eosinophils, lymphocytes and monocytes.

\subsubsection{Statistical analysis}

Data were grouped and presented as means and standard deviations. Normality and homoscedasticity of the data were verified. The significance of differences among groups was assessed by analysis of variance (ANOVA) followed by post-hoc Tukey test. The significance level was set at $5 \%$ for all tests.

\subsection{Experiment 2-Bacillus species in the treatment of EP}

In Experiment 2, the effects of Bacillus species as adjuncts to SRP for the treatment of EP were analyzed. 24 rats were randomly assigned to one of four experimental groups $(n=6)$ : C2 (control), PROB2 (probiotic), EP-SRP2 (experimental periodontitis + SRP) and EP-SRP-PROB2 (experimental periodontitis + SRP + probiotic).

\subsubsection{Induction of EP, SRP, probiotic therapy and euthanasia}

In the beginning of the experiment (day 0), the animals of Groups EP-SRP2 and EP-SRP-PROB2 were submitted to EP induction, as described in Experiment 1. After 14 days, all animals were anesthetized as described in the EP induction procedure. Then, in Groups EP-SRP2 and EP-SRP-PROB2, the ligatures were removed and the right mandibular first molars were subjected to SRP with manual Mini Five 1-2 curettes (Hu-Friedy Co. Inc., Chicago, IL, USA) through ten distal-mesial traction movements on the buccal and lingual aspects. The furcation and interproximal areas were scaled with the same curettes through cervico-occlusal traction movements. SRP procedures were performed by the same 
experienced and blinded operator (L.F.F.O.). Animals of Groups $\mathrm{C} 2$ and PROB2 were not subjected to EP induction.

For Groups PROB2, the same probiotic therapy described in Experiment 1 was administered for 15 days, starting immediately after SRP in Group EP-SRP-PROB2. Animals of Groups C2 and EP2 received water not supplemented with probiotic.

The animals were euthanized at day 29 with a lethal dose ( $150 \mathrm{mg} / \mathrm{kg}$ body weight) of sodium thiopental (Thiopentax ${ }^{\mathbb{R}}$, Cristalia Produtos Quimicos Farmaceuticos Ltda., Sao Paulo, SP, Brazil). The hemimandibles were excised and fixed in $4 \%$ formaldehyde for $48 \mathrm{~h}$ in preparation for histomorphometric and immunohistochemical analyses.

\subsubsection{Histopathological and histometric analyses}

The right hemimandibles were decalcified in 4\% EDTA solution. After complete decalcification, they were processed and embedded in paraffin. Serial sections, $4 \mu \mathrm{m}$ thick, were obtained in a mesiodistal direction. The sections were stained with hematoxylin and eosin for analysis by light microscopy. The histopathological analysis was performed by a blinded and certified histologist (E.E.).

For histometric analysis, two sections representing the most central buccal-lingual portion in the furcation area of the right mandibular first molars were selected. The images of the histologic sections were captured by a digital camera (DFC295, Leica Microsystems, Wetzlar, Germany) connected to a light microscope (DM2000, Leica Microsystems, Wetzlar, Germany). The digital images were saved on a computer and then analyzed using appropriate software (Diracon Bio Informatica Ltda., Vargem Grande do Sul, SP, Brazil). The furcation area not filled with bone or periodontal ligament (Area of No Bone or Periodontal Ligament-ANBL) was measured by outlining the region surrounded by the roof of the furcation (RF), the most coronal portion of the $A B C$ in the furcation, the mesial and the distal roots of the first molar. In order to assess the attachment loss (AL), the linear distance between the CEJ and the epithelial attachment was measured on the distal root of the right mandibular first molar. The values of ANBL and AL for each animal were represented by the mean values of the two histologic sections. Histometric analysis was performed by one calibrated examiner (C.G.S.) who was blinded to the experimental groups and treatments rendered.

\subsubsection{Immunohistochemical analyses}

Immunohistochemical processing was performed using the indirect immunoperoxidase method, as previously described (Furlaneto et al., 2014). The histological slides containing samples from all experimental groups were incubated with one of the following primary antibodies: rabbit anti-interleukin-1 beta-IL$1 \beta$ (1:100-Rabbit anti-IL-1 $\beta$-SC 7884, Santa Cruz Biotechnology, Santa Cruz, CA, USA), goat anti-interleukin-10-IL-10 (1:100-Goat anti-IL-10-SC 1783, Santa Cruz Biotechnology, Santa Cruz, CA, USA) or goat anti-tartrate-resistant acid phosphatase (TRAP; 1:100-Goat anti-TRAP-SC 30833, Santa Cruz Biotechnology, Santa Cruz, CA, USA).

A quantitative immunolabeling analysis was performed for TRAP-positive cells. TRAP-positive cells were counted in an area of $2000 \mu \mathrm{m} \times 2000 \mu \mathrm{m}$ in the central part of furcation septum at $200 \times$ magnification. The coronal limit of the area was the $A B C$, which extended apically for a distance of $2000 \mu \mathrm{m}$.

Semi-quantitative immunolabeling analyses of IL-1 $\beta$ and IL-10 were conducted in the entire furcation region at $400 \mathrm{x}$ magnification. The following scores were determined: score $0-$ no immunolabeling (total absence of immunoreactivity in the area);
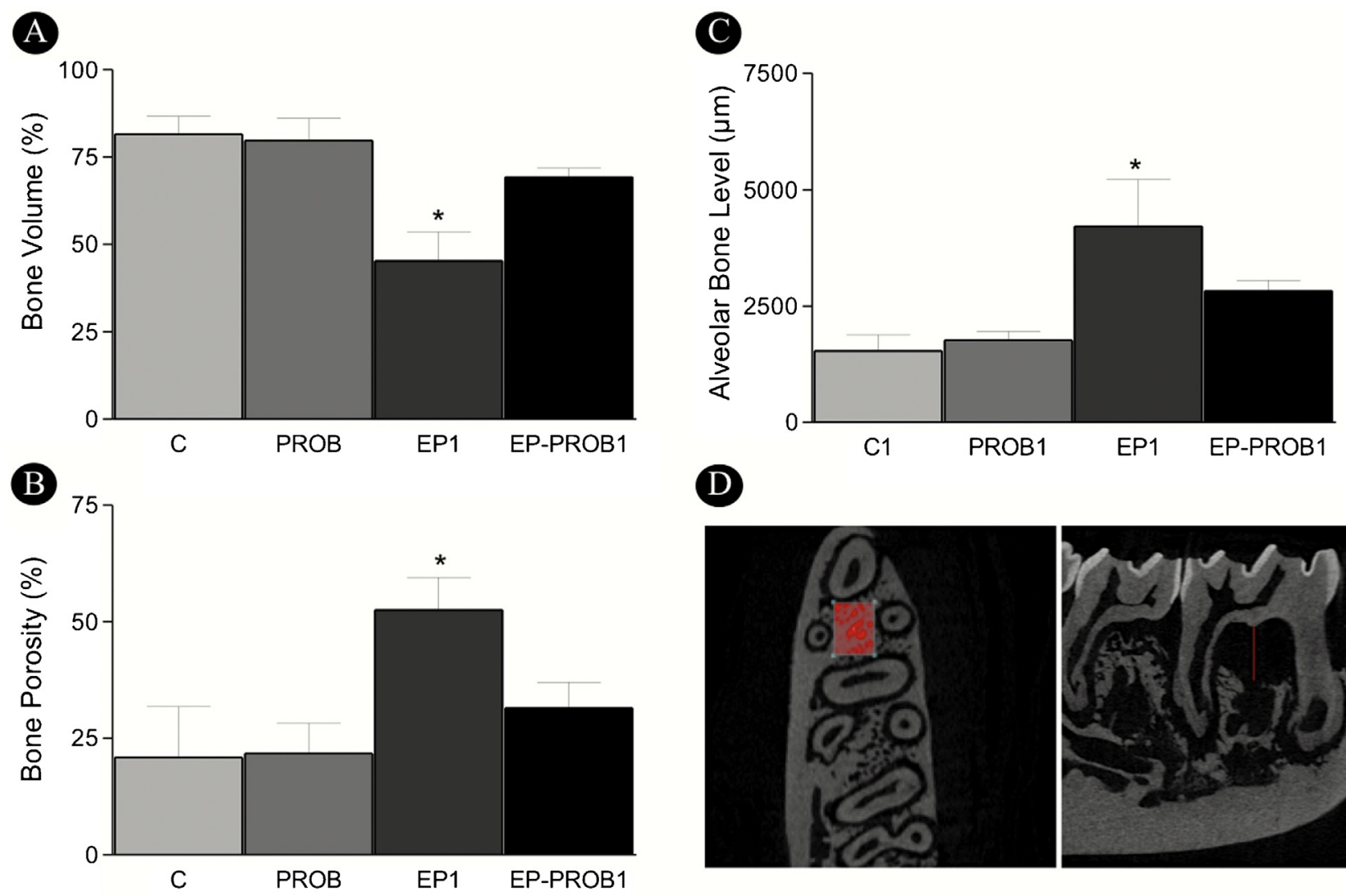

D)
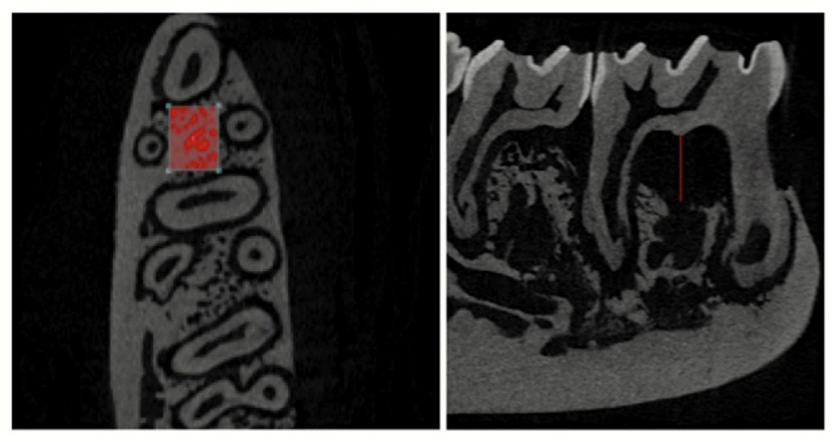

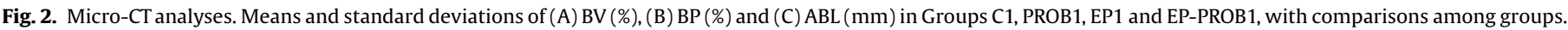

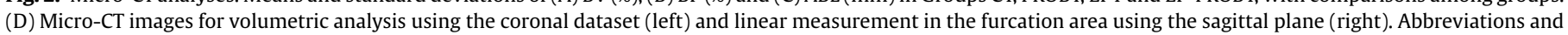
symbols: $\mathrm{BV}=$ bone volume; $\mathrm{BP}=$ bone porosity; $\mathrm{ABL}=$ alveolar bone level; ${ }^{*}=$ significant difference when compared with all the other groups $(P<0.05)$. 
score 1 -low immunolabeling pattern $\left(\approx^{1 / 3}\right.$ of the area presenting immunoreactivity); score 2-moderate immunolabeling pattern $\left(\approx^{2} / 3\right.$ of the area presenting immunoreactivity) and score 3 -high immunolabeling pattern (almost the entire area presenting immunoreactivity).

Histologic sections were evaluated under light microscopy with an optical microscope (Axiovision 4.8.2, Carl Zeiss MicroImaging $\mathrm{GmbH}$, Jena, Germany) by one calibrated examiner (E.E.) who was blinded to the experimental groups and treatments rendered.

\subsubsection{Statistical analysis}

Data were grouped and presented as means and standard deviations (continuous variables) or medians, interquartiles ranges and maximum and minimum values (ordinal variables). Normality and homoscedasticity of the data were verified. The significance of differences among groups in relation to the immunolabeling pattern of IL-1 $\beta$ and IL-10 was determined by Kruskal-Wallis tests, followed by Dunn's multiple comparison post-test. The data from the histometric analysis were assessed by ANOVA followed by posthoc Tukey test. The significance level was set at $5 \%$ for all tests.

\section{Results}

3.1. Experiment 1-Bacillus species in the development of EP

\subsubsection{Micro-CT analyses}

Group EP-PROB1 presented greater BV and decreased ABL and BP when compared with Group EP1 $(P<0.05)$. Means and standard deviations of $\mathrm{ABL}, \mathrm{BV}$ and $\mathrm{BP}$, as well as intergroup comparisons, are depicted in Fig. 2.

\subsubsection{Western blot analysis}

Group EP1 presented RANKL/OPG ratio significantly greater than Groups $\mathrm{C} 1$ and PROB1 $(P<0.05$; Fig. 3A). No significant differences regarding RANKL/OPG ratio were observed among Groups C1, PROB1 and EP-PROB1 or between Groups EP1 and EPPROB1 $(P>0.05$; Fig. 3A).
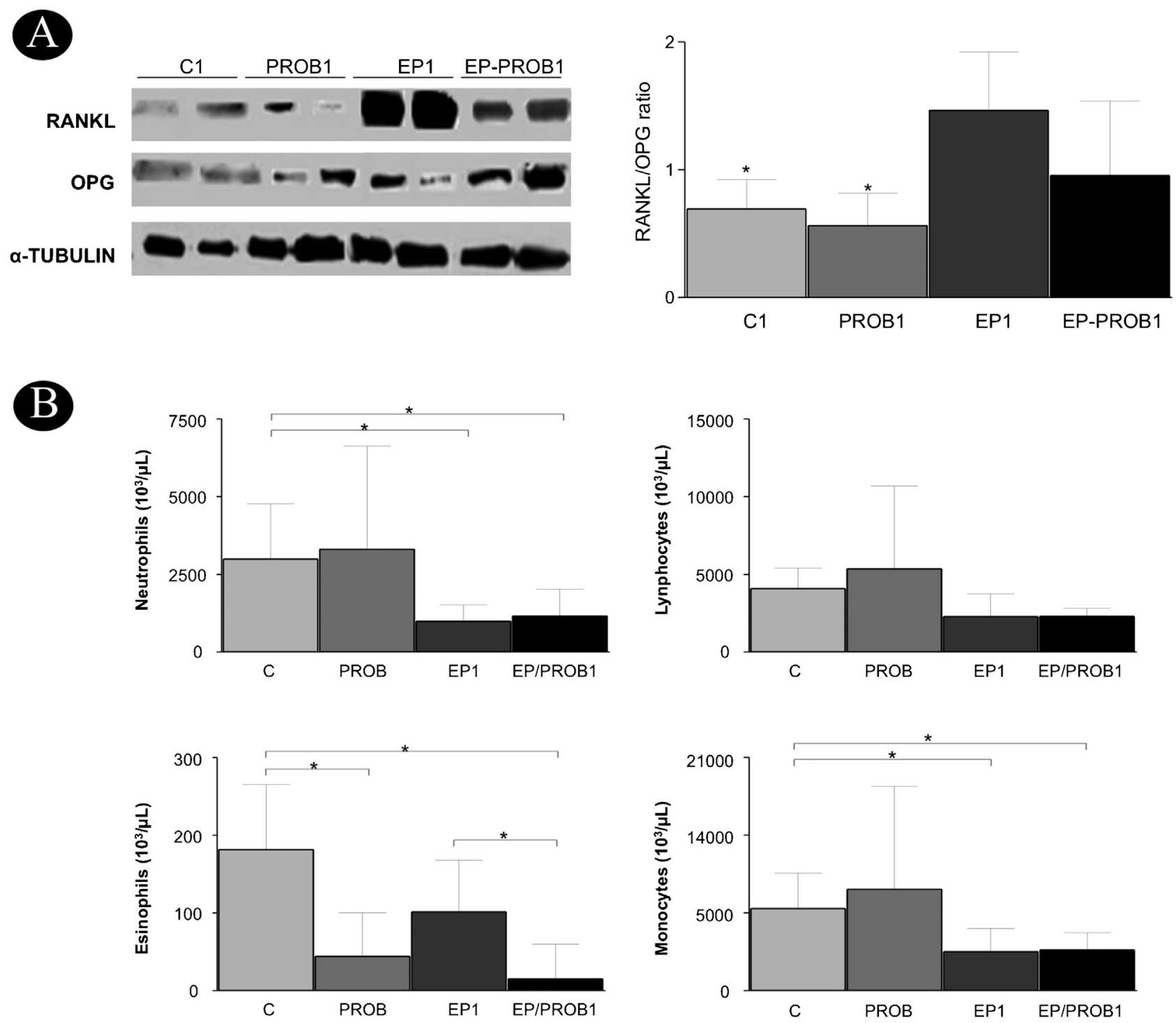

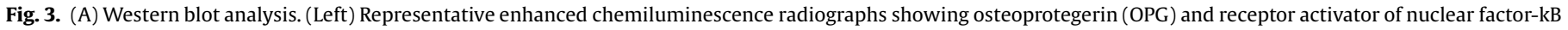

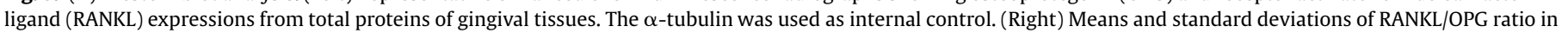

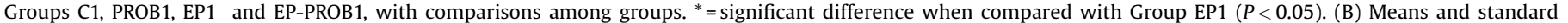

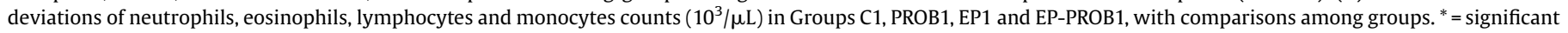
difference between groups $(P<0.05)$. 


\subsubsection{Hematological analysis}

Groups EP1 and EP-PROB1 presented a significantly reduced quantity of neutrophils and monocytes compared to Group C1 $(P<0.05$; Fig. 3B). No significant differences regarding the number of neutrophils, lymphocytes or monocytes were observed between Groups EP1 and EP-PROB1 ( $P>0.05$; Fig. 3B). A decreased number of eosinophils was observed in Groups PROB1 and EP-PROB1 in relation to Group C1 $(P<0.05$; Fig. 3B). Group EP1 presented a significantly greater quantity of eosinophils than Group EP-PROB1 $(P<0.05$; Fig. 3B).

\subsection{Experiment 2-Bacillus species in the treatment of EP}

\subsubsection{Histopathological analysis}

In Groups C2 and PROB2, periodontal ligament (PL) presented a great amount of collagen fibers, fibroblasts and
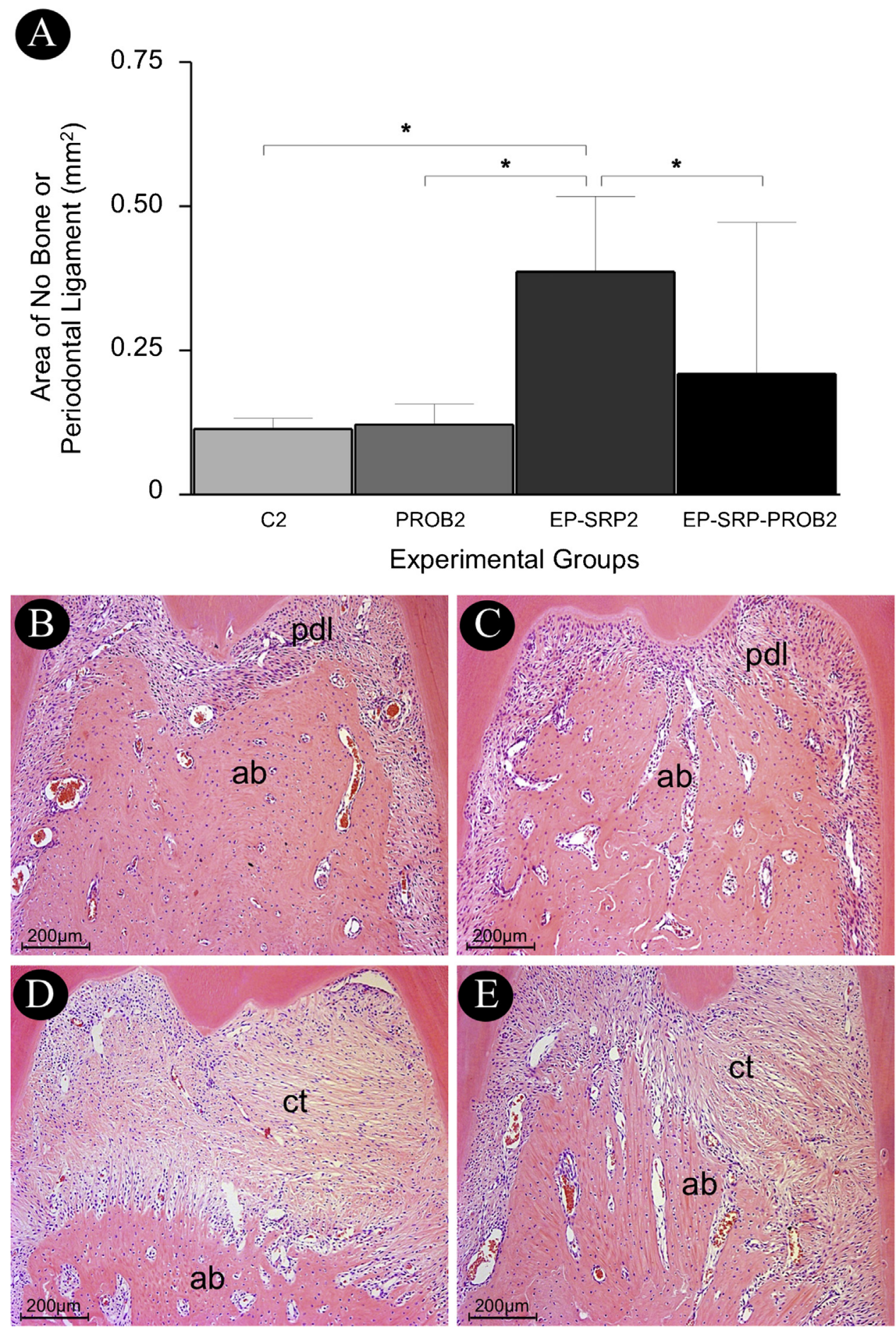

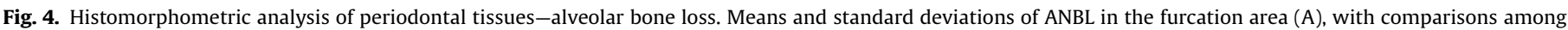

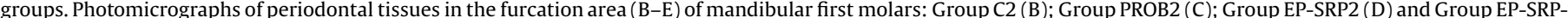

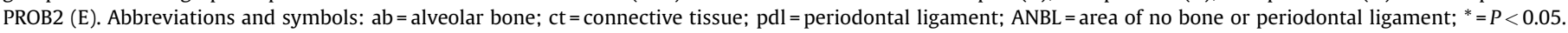
Scale bars: $B-E=200 \mu \mathrm{m}$. (Hematoxylin and Eosin stain). 
blood vessels. Collagen fibers were inserted both in cementum and in alveolar bone. The cementum surface was totally sound and covered with cementoblasts. The bone tissue of the furcation region presented a few irregularities on its surface and it was coated with osteoblasts or bone lining cells (Fig. 4B and C). In both groups, junctional and sulcular epithelia were completely intact in the interproximal area (Fig. 5B and C).
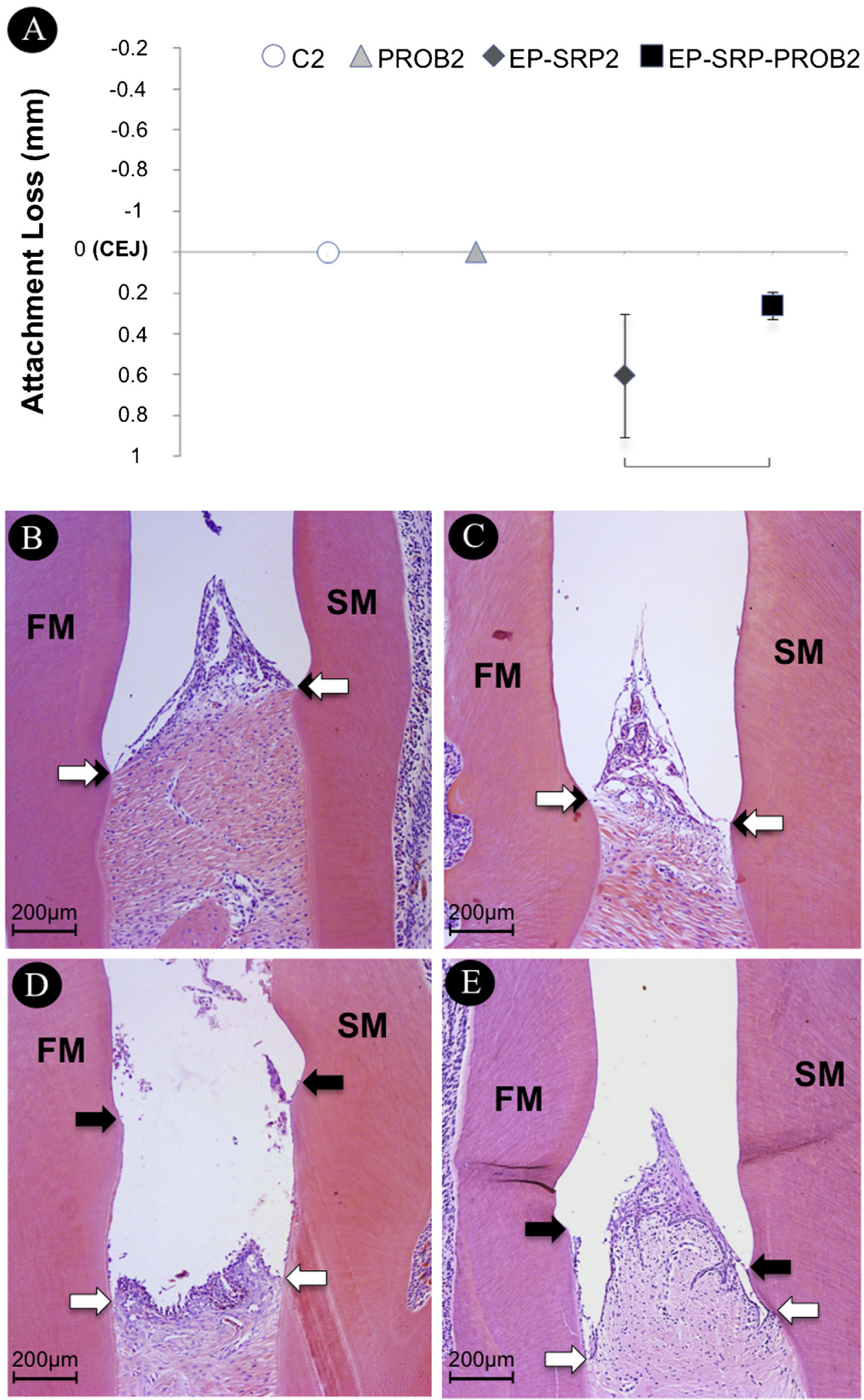

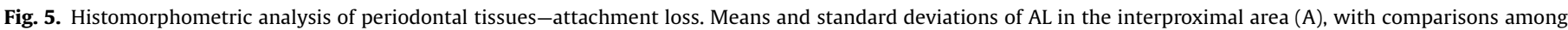

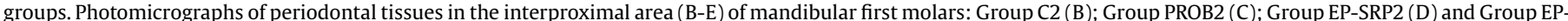

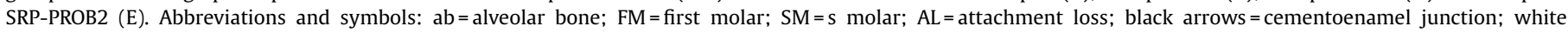
arrows $=$ epithelial attachment; ${ }^{*}=P<0.05$. Scale bars: B-E $=200 \mu \mathrm{m}$. (Hematoxylin and Eosin stain). 
In Group EP-SRP2, the bone of the interradicular septum presented irregular outer contours and numerous active osteoclasts. The connective tissue presented a moderate inflammatory infiltrate consisting primarily of neutrophils (Fig. 4D). A severe damage of the junctional and sulcular epithelia was observed in the interproximal area (Fig. 5D). In Group EP-SRP-PROB2, the inflammatory infiltrate in the furcation region was less extensive than the one observed in Group EP-SRP2 and was mainly composed of neutrophils. The connective tissue adjacent to the interradicular septum contained a moderate number of fibroblasts and collagen fibers, a large number of blood vessels and few inflammatory cells. The alveolar bone crest was positioned more coronally when compared with the one of Group EP-SRP2 (Fig. 4E) and few active osteoclasts were observed. The junctional and sulcular epithelia of the interproximal area were less damaged than that of Group EP-SRP2 (Fig. 5E).

\subsubsection{Histometric analysis}

Group EP-SRP-PROB2 showed decreased ANBL (Fig. 4A) and AL (Fig. 5A; $P<0.05$ ) when compared with Group EP-SRP2. No
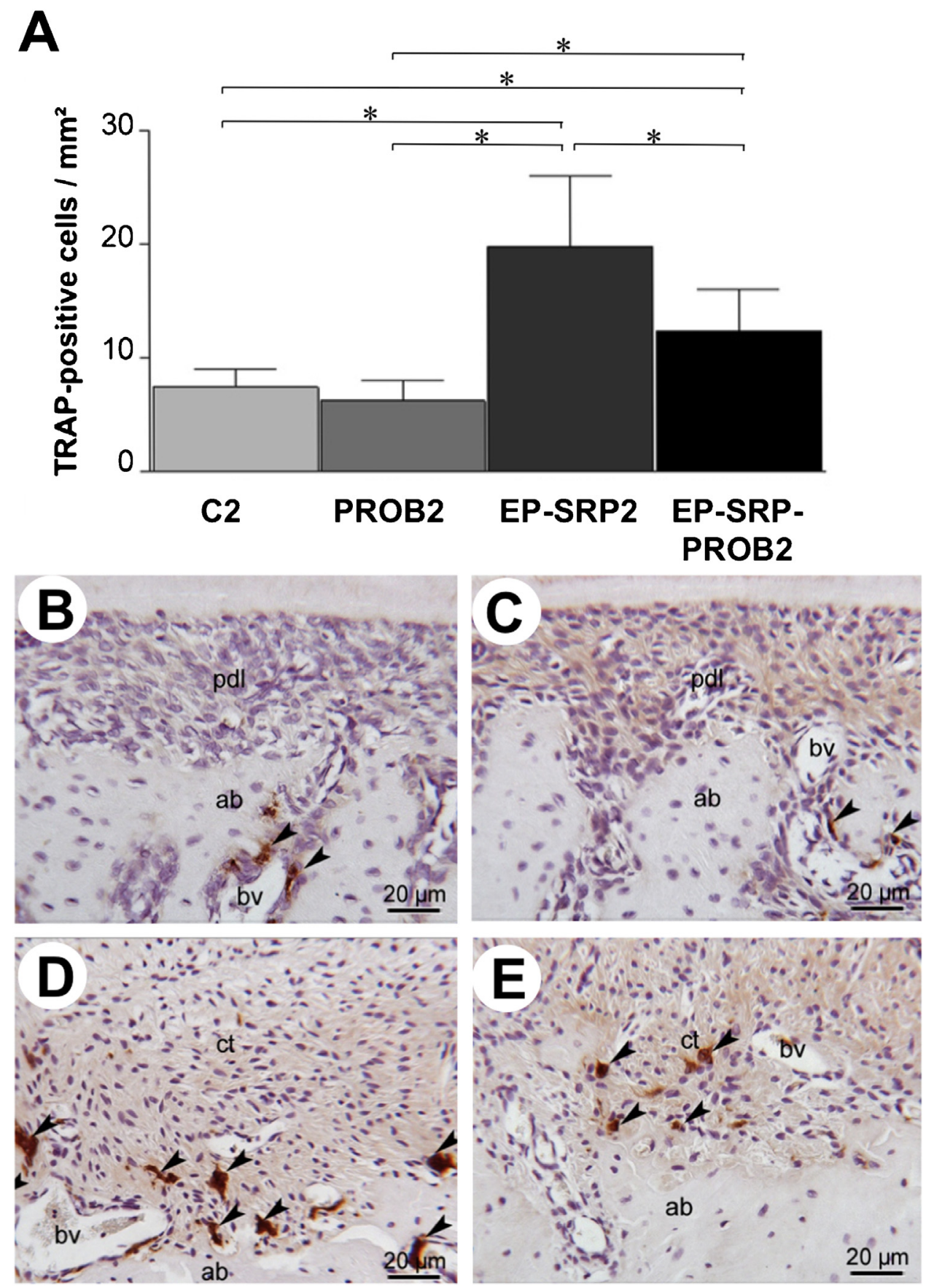

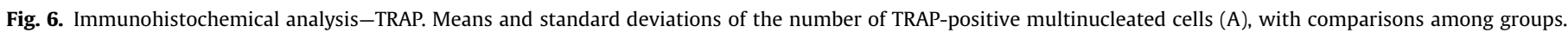

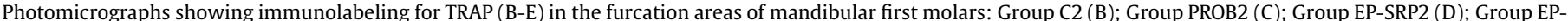

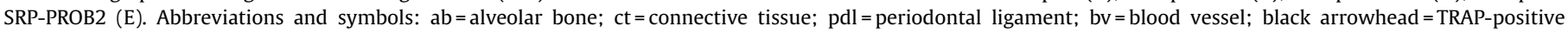
multinucleated cell; ${ }^{*}=$ statistically significant difference (ANOVA, $P<0.05$ ). Scale bars: $\mathrm{B}-\mathrm{E}=20 \mu \mathrm{m}$. (Hematoxylin counterstaining). 
differences in ANBL were observed among Groups C2, PROB2 and EP-SRP-PROB2. Groups C2 and PROB2 did not present AL.

\subsubsection{Immunohistochemical analyses}

Group EP-SRP-PROB2 presented less TRAP-positive multinucleated cells with decreased immunolabeling for IL-1 $\beta$ and increased immunolabeling for IL-10 than Group EP-SRP2 $(P<0.05$; Figs. 6 and 7).

\section{Discussion}

The use of Bacillus species as probiotic dietary supplements is expanding rapidly with an increasing number of studies demonstrating immune stimulation, antimicrobial activities and competitive exclusion (Cutting, 2011). In this study, two independent experiments, consisting of different analyses, were performed in order to investigate the effects of Bacillus species on the development and treatment of EP. In Experiment 1, probiotic therapy reduced alveolar bone loss and decreased the number of peripheral blood eosinophils. Furthermore, in Experiment 2, the therapy provided additional benefits to SRP in the treatment of periodontitis, reducing the number of osteoclasts, as well as the loss of attachment and alveolar bone.

In Experiment 1, EP promoted bone resorption in Groups EP1 and EP-PROB1. However, the damage was minimized by the treatment with probiotic. In Group EP-PROB1, the oral administration of $B$. subtilis and B. licheniformis led to an increase of $52.74 \%$ in BV and to decreases of $40.08 \%$ in BP and $66.97 \%$ in ABL when compared with Group EP1. These changes were observed using microtomographic analyses. These results may be explained, in part, by the effects of the probiotic therapy on the hematologic parameters and the RANKL/OPG ratio in periodontal tissues.

Group EP1 presented a significantly greater number of peripheral blood eosinophils than Group EP-PROB1. Eosinophils play a role as antigen-presenting cells that modulate immune responses by amplifying T-helper cell type 2 (Th2) responses (Padigel, Lee, Nolan, Schad, \& Abraham, 2006; Shi, 2004; Spencer \& Weller, 2010) therefore perpetuating the inflammatory process (Svensson \& Wenneras, 2005). In this context, it can be suggested that the probiotic therapy inhibited Th2 response in Group EPPROB1, decreasing the severity and progression of EP in these animals. This hypothesis is supported by some studies that have
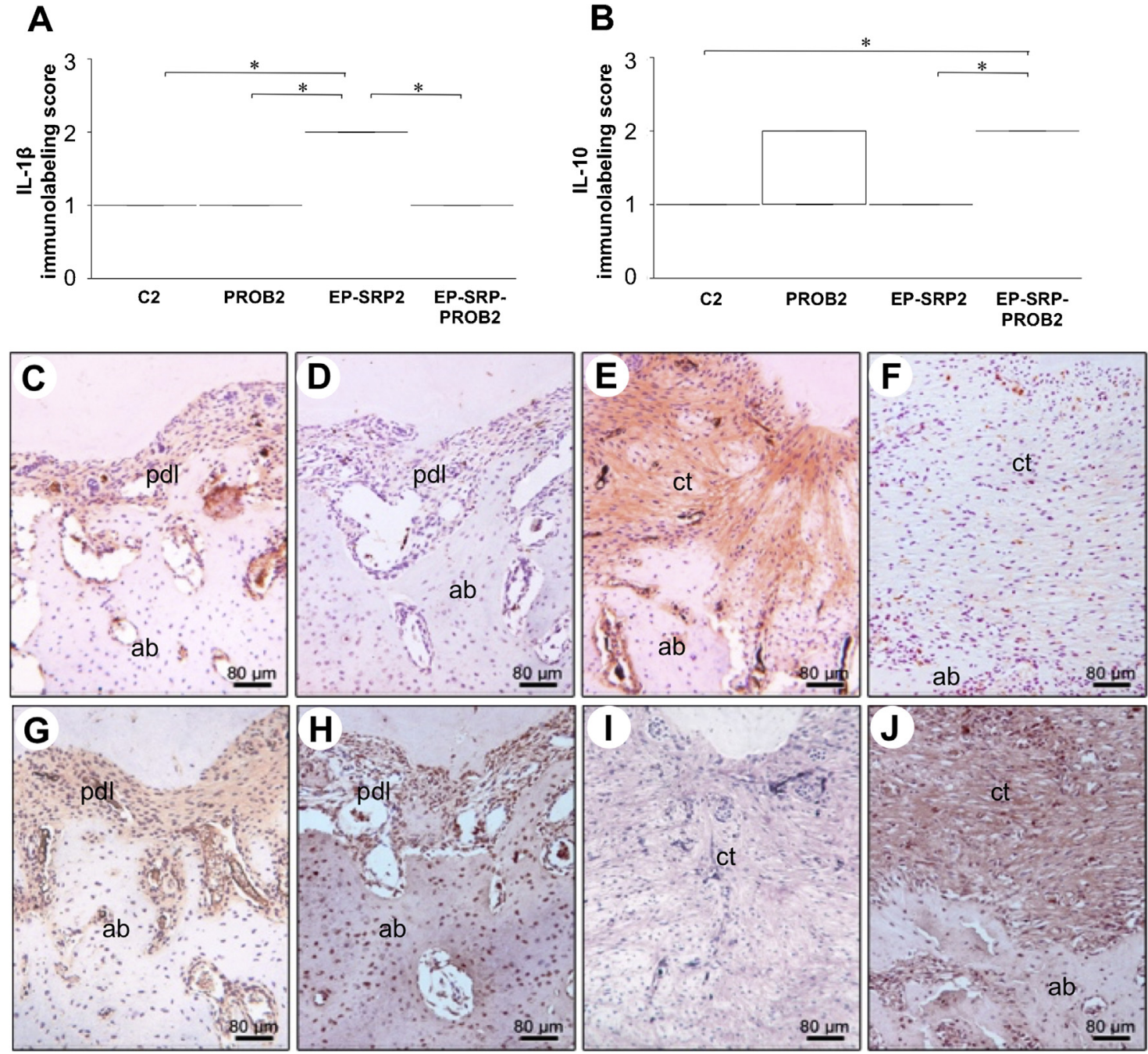

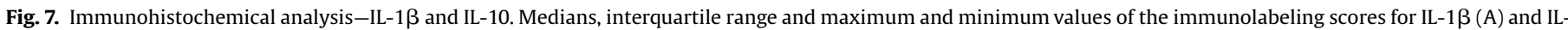

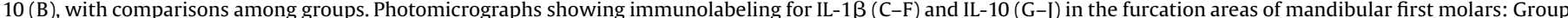

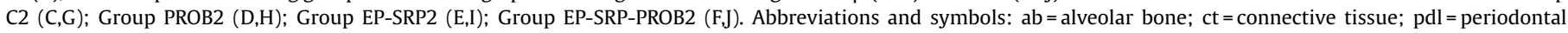
ligament; ${ }^{*}=$ statistically significant difference (Kruskal-Wallis, $P<0.05$ ). Scale bars: $\mathrm{C}-\mathrm{J}=80 \mu \mathrm{m}$. (Hematoxylin counterstaining). 
already demonstrated that $B$. subtilis-derived poly-c-glutamic acid (cPGA) is capable of inhibiting Th2 Lee, Park, Park, and Hong (2013) and Th17 responses (Lee, Kim, Won, Lee, Sung, \& Poo, 2014). cPGAtreated mice exhibited reductions in both frequencies and absolute numbers of splenic mast cells, basophils and eosinophils (Lee et al., 2013). In the present study, it seems that EP did not promote a significant increase in the number of eosinophils in Group EP1 when compared with Group C1. A possible explanation for that would be the release of small but consistent amounts of chemotactic factors into the circulation in patients with periodontitis and consequent eosinophil accumulation at tissue sites (e.g. lymph nodes), reducing the number of circulating eosinophils (Lopez, Loos, \& Baelum, 2012). It is also important to emphasize that, although some studies have documented differences in the hematological parameters for subjects with chronic periodontitis compared to healthy subjects, the findings are not always conclusive (Lopez et al., 2012). The variation found in the blood parameters investigated in adult study populations frequently appears to be modest and the values frequently do not exceed the normal reference values (Loos, 2005).

Group EP1 was the only one to present a RANKL/OPG ratio, which demonstrates the effects of EP on alveolar bone resorption, significantly greater than that of Groups C1 and PROB1. RANKL is a key molecule in osteoclast activation and OPG is a decoy receptor for RANKL. Therefore, the increase in RANKL/OPG ratio favors bone resorption through osteoclastogenesis and activation of osteoclasts (Kajiya et al., 2010). In contrast to what was observed in Group EP1, no significant differences in RANKL/OPG ratio were observed when Group EP-PROB1 was compared with Groups $\mathrm{C} 1$ and $\mathrm{PROB} 1$. This finding may be explained in part by the possible effects of Bacillus species products on the reduction of interleukin (IL)-17 levels (Lee et al., 2014). IL-17A can play a role in the pathogenesis of periodontitis by modulating bone remodeling through induction of RANKL (Awang et al., 2014; Kotake, Yago, Kawamoto, \& Nanke, 2012). Besides, it has been shown that serum levels of IL-17A correlate with clinical attachment loss in patients with aggressive periodontitis (Schenkein et al., 2010). It is also important to consider that Bacillus species increased the expression of OPG in periodontal tissues in a previous study by this group (Foureaux et al., 2014).

In Experiment 2, probiotic administration started immediately after SRP in an attempt to improve the impact of the adjunctive therapy. According to Teughels et al. (2011) probiotics will have difficulty colonizing the mouth and exerting beneficial clinical effects in an oral microbiological environment that has already matured. It is important to consider that the efficacy of SRP in this experimental model has been clearly demonstrated (Garcia et al., 2015; Theodoro et al., 2015). In these studies, the animals treated with SRP after EP induction had a significant reduction in alveolar bone loss compared to untreated animals (Garcia et al., 2015; Theodoro et al., 2015). Significant reductions in ANBL and AL (45.80\% and 56.69\%, respectively) were observed in Group EP-SRP-PROB2 when compared with Group EP-SRP2. These results demonstrate the potential of Bacillus species as adjuncts to SRP for the treatment of EP. It is possible that they can reduce the necessity for retreatment of periodontal sites in non-surgical approaches, as demonstrated by Teughels et al. (2013) when the effects of Lactobacillus bacteria as an adjunct to SRP were evaluated. According to Sbordone, Ramaglia, Gulletta, and Iacono, (1990) SRP presents short-term effects and the recolonization of treated pockets by potential periodontal pathogens can be observed 3 weeks post-SRP. In this context, a hypothesis to explain the results of the present study is that Bacillus species reduced the recolonization of treated periodontal pockets by periodontopathogenic microorganisms. Furthermore, the Bacillus species might have modulated the immunoinflammatory response of the animals, improving tissue repair after SRP procedures.

Regarding immunomodulatory properties, it has been demonstrated that $B$. subtilis can reduce pro-inflammatory cytokines (IL-1, tumor necrosis fator-alpha [TNF- $\alpha$ ], IL-6 and interferon-gamma) and increase anti-inflammatory cytokines (IL-10 and transforming growth factor- $ß$ ) in the plasma of patients with inflammatory bowel disease (Selvam et al., 2009). To the authors' knowledge, there are no studies evaluating the immunoinflammatory properties of Bacillus species in periodontal tissues. In the present study, it was observed that Bacillus species influenced the balance of proinflammatory and anti-inflammatory cytokines. A lower expression of IL-1 $\beta$ and a greater expression of IL-10 were observed in Group EP-SRP-PROB2 when compared with Group EP-SRP2. IL10 exerts inhibitory effects on IL- $1 \beta$ and TNF- $\alpha$, whose synergistic actions on inflammatory processes amplify the host response (Moore, de Waal Malefyt, Coffman, \& O'Garra, 2001). The elevated ratio IL-1 $\beta /$ IL-10 in gingival crevicular fluid may be related to progression of aggressive periodontitis (Teles et al., 2010). Considering the antimicrobial properties, compounds of Bacillus species have been used in agriculture for the inhibition of phytopathogens, for the stimulation of plant growth and in the food industry as probiotics (Mongkolthanaruk, 2012). There is only one study that reported the effects of Bacillus species on oral biofilm. Tsubura et al. (2009) verified that a mouth rinse prepared from the supernatant of a cultured medium of $B$. subtilis promoted a marked change in the scores of BANA test in patients with chronic periodontitis. In this study, neither the microbiologic profile of the oral biofilm of the animals, nor the colonization of their oral cavity by the probiotic bacteria administered were evaluated. Nevertheless, it was observed that Bacillus species present antimicrobial activity against some periodontal pathogens in in vitro assays performed in the laboratory of this group (data not shown). Regarding the colonization of the oral cavity, it is important to consider that many of the gastro-intestinal probiotics exert their effect without colonizing or with only a temporary colonization of the host (Teughels et al., 2011). Furthermore, probiotics may influence the biofilm not only quantitatively but also qualitatively. For example, Nissen, Sgorbati, Biavati, and Belibasakis (2014) demonstrated that lactobacilli can reduce the virulence of putative opportunistic oral pathogens. In this study, $L$. salivarius and $L$. gasseri down-regulated Aggregatibacter actinomycetemcomitans exotoxins expression (Nissen et al., 2014).

A decreased quantity of TRAP-positive cells was observed in Group EP-SRP-PROB2 when compared with Group EP-SRP2. It can be explained by the possible effects of Baccilus species and their subproducts on osteoclastic differentiation and activation paths, such as nuclear factor-kappa B (NF-kB) and mitogen-activated protein kinase (MAPK) pathways. Park, Kim, Kim, Ryu, and Lee (2010) demonstrated that surfactin produced by B. subtilis significantly reduces the production of TNF- $\alpha$, IL-1ß, IL-6 and IL12 through suppression of NF-kB activity in Porphyromonas gingivalis lipopolysaccharides-stimulated THP-1 human macrophage cells. In a previous study by this group (Foureaux et al., 2014), it was demonstrated that Bacillus species can decrease the expression of p38 MAPK in the periodontal tissues of stressed rats. According to Matsumoto, Sudo, Saito, Osada, \& Tsujimoto (2000) the activation of $\mathrm{p} 38$ MAPK pathway plays an important role in RANKL-induced osteoclastic differentiation of precursor bone marrow cells.

Most probiotic strains with reported beneficial effects in either animal or clinical studies are lactobacilli or bifidobacteria. However, many other bacterial genera have demonstrated probiotic potential, including spore-forming Bacillus species (Foligne et al., 2012; Hong, Duc le, \& Cutting, 2005; Sorokulova et al., 2008). The present study provided a proof-of-concept that 
$B$. subtilis and $B$. licheniformis present therapeutic potential in the prevention and treatment of periodontitis. Whether this probiotic preparation can find application as an adjunctive therapy in human periodontitis is yet to be investigated in clinical trials.

\section{Conclusions}

It can be concluded that the supplementation with Bacillus species provided a protective effect against bone loss and enhanced the effects of SRP in the treatment of EP in rats.

\section{Conflict of interest}

The authors declare that they do not have a conflict of interest.

\section{Funding}

Chr. Hansen (Hørsholm, Denmark) donated the probiotics. There is no relationship between any author and commercial firms that may pose a conflict of interest.

\section{Ethical approval}

The present study was conducted only after review and approval by the Ethics Committees on Animal Experimentation (ECAE) at School of Dentistry of Ribeirao Preto, University of São Paulo-FORP/USP (protocol 2013.1.993.58.6), at Federal University of Lavras-UFLA (protocol 036/2010) and at Lavras University Center-UNILAVRAS (protocol 012/2010).

\section{Acknowledgements}

The authors thank the National Council for Research and Technological Development (CNPq - Brasília, DF, Brazil, Process 475468/2010-4 - scholarship to L.J.P and Process 303555/2013-0scholarship to M.H.N.). The authors also thank Dr. Stephen Enrico Fucini (Division of Periodontics, Department of Surgery and Integrated Clinic, Dental School of Araçatuba, Univ. Estadual Paulista-UNESP, Araçatuba, Sao Paulo, Brazil) for language help during the prepare of this manuscript.

\section{References}

Awang, R. A., Lappin, D. F., MacPherson, A., Riggio, M., Robertson, D., Hodge, P., et al. (2014). Clinical associations between IL-17 family cytokines and periodontitis and potential differential roles for IL-17A and IL-17E in periodontal immunity. Inflammation Research, 63(12), 1001-1012.

Bengmark, S. (2001). Pre-, pro- and synbiotics. Current Opinion in Clinical Nutrition and Metabolic Care, 4(6), 571-579.

Bron, P. A., van Baarlen, P., \& Kleerebezem, M. (2012). Emerging molecular insights into the interaction between probiotics and the host intestinal mucosa. Nature Reviews Microbiology, 10(1), 66-78.

Cutting, S. M. (2011). Bacillus probiotics. Food Microbiology, 28(2), 214-220.

FAO/WHO, 2002. Guidelines for the Evaluation of Probiotics in Food. [cited 2015 August 14]; Available from: ftp://ftp.fao.org/es/esn/food/wgreport2.pdf.

Foligne, B., Peys, E., Vandenkerckhove, J., Van Hemel, J., Dewulf, J., Breton, J., et al. (2012). Spores from two distinct colony types of the strain Bacillus subtilis PB6 substantiate anti-inflammatory probiotic effects in mice. Clinical Nutrition (Edinburgh, Scotland), 31(6), 987-994.

Foureaux, R. de C., Messora, M. R., de Oliveira, L. F., Napimoga, M. H., Pereira, A. N., Ferreira, M. S., et al. (2014). Effects of probiotic therapy on metabolic and inflammatory parameters of rats with ligature-induced periodontitis associated with restraint stress. Journal of Periodontology, 85(7), 975-983.

Furlaneto, F. A., Nunes, N. L., Oliveira Filho, I. L., Frota, N. P., Yamamoto, K. O., Lisboa, M. R., et al. (2014). Effects of locally administered tiludronic acid on experimental periodontitis in rats. Journal of Periodontology, 85(9), 1291-1301.

Garcia, V. G., Novaes, V. C., de Almeida, J. M., Longo, M., Ervolino, E., Bomfim, S. R., et al. (2015). Evaluation of the progression and treatment of experimental periodontitis in rats subjected to chemotherapy with 5-fluorouracil. Supportive Care in Cancer, 23(7), 2007-2017.
Hong, H. A., Duc le, H., \& Cutting, S. M. (2005). The use of bacterial spore formers as probiotics. FEMS Microbiology Reviews, 29(4), 813-835.

Kajiya, M., Giro, G., Taubman, M. A., Han, X., Mayer, M. P., \& Kawai, T. (2010). Role of periodontal pathogenic bacteria in RANKL-mediated bone destruction in periodontal disease. Journal of Oral Microbiology, 2, 5532.

Kotake, S., Yago, T., Kawamoto, M., \& Nanke, Y. (2012). Role of osteoclasts and interleukin-17 in the pathogenesis of rheumatoid arthritis: crucial 'human osteoclastology'. Journal of Bone and Mineral Metabolism, 30(2), 125-135.

Lee, S. W., Park, H. J., Park, S. H., \& Hong, S. (2013). Oral administration of polygamma-glutamic acid prevents the development of atopic dermatitis in NC/Nga mice. Experimental Dermatology, 22(8), 561-563.

Lee, T. Y., Kim, D. J., Won, J. N., Lee, I. H., Sung, M. H., \& Poo, H. (2014). Oral administration of poly-gamma-glutamate ameliorates atopic dermatitis in $\mathrm{Nc}$ Nga mice by suppressing Th2-biased immune response and production of IL17A. The Journal of Investigative Dermatology, 134(3), 704-711.

Loos, B. G. (2005). Systemic markers of inflammation in periodontitis. Journal of Periodontology, 76(Suppl. 11), 2106-2115.

Lopez, R., Loos, B. G., \& Baelum, V. (2012). Hematological features in adolescents with periodontitis. Clinical Oral Investigations, 16(4), 1209-1216.

Matsumoto, M., Sudo, T., Saito, T., Osada, H., \& Tsujimoto, M. (2000). Involvement of p38 mitogen-activated protein kinase signaling pathway in osteoclastogenesis mediated by receptor activator of NF-kappa B ligand (RANKL). The Journal of Biological Chemistry, 275(40), 31155-31161.

Messora, M. R., Oliveira, L. F., Foureaux, R. C., Taba, M. Jr., Zangeronimo, M. G., Furlaneto, F. A., et al. (2013). Probiotic therapy reduces periodontal tissue destruction and improves the intestinal morphology in rats with ligatureinduced periodontitis. Journal of Periodontology, 84(12), 1818-1826.

Mongkolthanaruk, W. (2012). Classification of Bacillus beneficial substances related to plants, humans and animals. Journal of Microbiology and Biotechnology, 22 (12), 1597-1604.

Moore, K. W., de Waal Malefyt, R., Coffman, R. L., \& O'Garra, A. (2001). Interleukin10 and the interleukin-10 receptor. Annual Review of Immunology, 19, 683-765.

Nissen, L., Sgorbati, B., Biavati, B., \& Belibasakis, G. N. (2014). Lactobacillus salivarius and $L$. gasseri down-regulate Aggregatibacter actinomycetemcomitans exotoxins expression. Annals of Microbiology, 64, 614-617.

Padigel, U. M., Lee, J. J., Nolan, T. J., Schad, G. A., \& Abraham, D. (2006). Eosinophils can function as antigen-presenting cells to induce primary and secondary immune responses to Strongyloides stercoralis. Infection and Immunity, 74(6), 3232-3238.

Park, S. Y., Kim, Y. H., Kim, E. K., Ryu, E. Y., \& Lee, S. J. (2010). Heme oxygenase1 signals are involved in preferential inhibition of pro-inflammatory cytokine release by surfactin in cells activated with Porphyromonas gingivalis lipopolysaccharide. Chemico-biological Interactions, 188(3), $437-445$.

Sanz, M., \& van Winkelhoff, A. J. (2011). Periodontal infections: understanding the complexity?consensus of the Seventh European Workshop on Periodontology. Working Group 1 of Seventh European Workshop on Periodontology. Journal of Clinical Periodontology, 38(Suppl. 11), 3-6.

Sanz, M., \& Quirynen, M. (2005). Advances in the aetiology of periodontitis. Group A consensus report of the 5th European Workshop in Periodontology. European Workshop in Periodontology group A. Journal of Clinical Periodontology, 32 (Suppl. 6), 54-56.

Sbordone, L., Ramaglia, L., Gulletta, E., \& Iacono, V. (1990). Recolonization of the subgingival microflora after scaling and root planing in human periodontitis. Journal of Periodontology, 61(9), 579-584.

Schenkein, H. A., Koertge, T. E., Brooks, C. N., Sabatini, R., Purkall, D. E., \& Tew, J. G. (2010). IL-17 in sera from patients with aggressive periodontitis. Journal of Dental Research, 89(9), 943-947.

Selvam, R., Maheswari, P., Kavitha, P., Ravichandran, M., Sas, B., \& Ramchand, C. N. (2009). Effect of Bacillus subtilis PB6, a natural probiotic on colon mucosal inflammation and plasma cytokines levels in inflammatory bowel disease. Indian Journal of Biochemistry and Biophysics, 46(1), 79-85.

Shi, H. Z. (2004). Eosinophils function as antigen-presenting cells. Journal of Leukocyte Biology, 76(3), 520-527.

Sorokulova, I. B., Pinchuk, I. V., Denayrolles, M., Osipova, I. G., Huang, J. M., Cutting, S. M., et al. (2008). The safety of two Bacillus probiotic strains for human use Digestive Diseases and Science, 53(4), 954-963.

Spencer, L. A., \& Weller, P. F. (2010). Eosinophils and Th2 immunity: contemporary insights. Immunology and Cell Biology, 88(3), 250-256.

Stamatova, I., \& Meurman, J. H. (2009a). Probiotics and periodontal disease. Periodontology 2000, 51, 141-151.

Stamatova, I., \& Meurman, J. H. (2009b). Probiotics: health benefits in the mouth. American Journal of Dentistry, 22(6), 329-338.

Svensson, L., \& Wenneras, C. (2005). Human eosinophils selectively recognize and become activated by bacteria belonging to different taxonomic groups. Microbes and Infection/Institut Pasteur, 7(4), 720-728.

Teles, R. P., Gursky, L. C., Faveri, M., Rosa, E. A., Teles, F. R., Feres, M., et al. (2010). Relationships between subgingival microbiota and GCF biomarkers in generalized aggressive periodontitis. Journal of Clinical Periodontology, 37(4), 313-323.

Teughels, W., Loozen, G., \& Quirynen, M. (2011). Do probiotics offer opportunities to manipulate the periodontal oral microbiota? Journal of Clinical Periodontology, 38(Suppl. 11), 159-177.

Teughels, W., Durukan, A., Ozcelik, O., Pauwels, M., Quirynen, M., \& Haytac, M. C. (2013). Clinical and microbiological effects of Lactobacillus reuteri probiotics in 
the treatment of chronic periodontitis: a randomized placebo-controlled study. Journal of Clinical Periodontology, 40(11), 1025-1035.

Theodoro, L. H., Caiado, R. C., Longo, M., Novaes, V. C., Zanini, N. A., Ervolino, E., et al. (2015). Effectiveness of the diode laser in the treatment of ligature-induced periodontitis in rats: a histopathological, histometric, and

immunohistochemical study. Lasers in Medical Science, 30(4), 1209-1218.

Tsubura, S., Mizunuma, H., Ishikawa, S., Oyake, I., Okabayashi, M., Katoh, K., et al (2009). The effect of Bacillus subtilis mouth rinsing in patients with periodontitis. European Journal of Clinical Microbiology E' Infectious Diseases, 28 (11), 1353-1356.

Vivekananda, M. R., Vandana, K. L., \& Bhat, K. G. (2010). Effect of the probiotic Lactobacilli reuteri (Prodentis) in the management of periodontal disease: a preliminary randomized clinical trial. Journal of Oral Microbiology, 2, 5344.

Vuotto, C., Barbanti, F., Mastrantonio, P., \& Donelli, G. (2014). Lactobacillus brevis CD2 inhibits Prevotella melaninogenica biofilm. Oral Diseases, 20(7), 668-674. 